\title{
On lattices acting on boundaries of semi-simple groups
}

\author{
R. J. SPATZIER \\ Department of Mathematics, University of Maryland, College Park, MD 20742, USA
}

(Revised version received 4 January 1982)

Abstract. It is shown that for a lattice $\Gamma$ in a semi-simple group of real rank 1 the action on the boundary always admits an equivariant topological $\Gamma$-factor. We also show that there are no such factors for $\operatorname{SL}(n, \mathbb{Z})$ acting on $\mathbb{P}^{n-1}, n \geq 3$.

\section{Introduction}

In his proof of the finiteness theorem in [3] and [4] Margulis classifies all measurable $\Gamma$-quotients of the maximal boundary $G / P$ for an irreducible lattice $\Gamma$ in a semisimple Lie group $G$ (finite centre, no compact factors) of real rank at least two. In fact, he shows that they are all of the form $G / P^{\prime}$ for some parabolic subgroup $P^{\prime}$. At the same time his method allows one to 'construct' non-trivial SL $(2, \mathbb{Z})$-quotients of $S^{1}$ in the measurable sense. In fact, his method generalizes to some other rank 1 lattices.

At the end of his paper Margulis asks whether one could have topological (Hausdorff) quotients for SL $(n, \mathbb{Z})$ acting on $\mathbb{P}^{n-1}, n \geq 2$. R. Zimmer proved in [7] that, for $n>2$, any such quotient is trivial. Here we first propose a geometrical method to construct factors of the boundary for any lattice in a rank 1 group. Measure-theoretically though, these quotients will be trivial. Then we present another argument for the triviality for $n \geq 2$. For general $\Gamma$ and $G$ the question is still open. Our argument might essentially carry over to the case of split lattices.

\section{The rank 1 case}

To fix notation, let $G$ be a connected simple Lie group without compact factors of real rank 1 . Let $\Gamma$ be a lattice in $G$. $H$ will denote the globally symmetric space $G / K$ for $K$ a maximal compact subgroup of $G$. $B$ will be the boundary of $H$. We shall use the geometric interpretation of [2] for $B$, i.e. $B$ is the set of equivalence classes of asymptotic geodesics. Finally, $M$ will be the locally symmetric space $\Gamma \backslash G / K$.

We start with a closed geodesic $\bar{\alpha}$ in $M$. Pick a covering geodesic $\alpha$ in $H$ and an axial isometry $\gamma$ for $\alpha$, i.e. $\gamma$ translates $\alpha$ into itself (cf. [2, § 6]). We identify a geodesic with its pair of endpoints in $B$. Let $(x, y)$ be the endpoints of $\alpha$. Suppose that $\gamma_{n}(x, y)$ converges to a pair of distinct points $(s, t)$ for some $\gamma_{n}$ in $\Gamma$ (in the cone topology) (cf. $[2, \S 2]$ ) and let $\beta$ be the geodesic joining $s$ to $t$. Then any point on $\beta$ is a limit point of points on the $\gamma_{n} \alpha$ 's. Since $\bar{\alpha}$ is closed, its pre-image in $H$ 
is closed and hence $\beta \in \Gamma \alpha$. Since $\Gamma$ is countable, there are at least two points on $\beta$ that lie on the same $\delta \alpha$ for some $\delta$ in $\Gamma$. By uniqueness of geodesics in negative curvature,

$$
\beta=\delta \alpha .
$$

We summarize our discussion in:

LEMMA 1. The orbit $\Gamma(x, y)$ is closed in $B \times B$-diagonal.

Consider the equivalence relation $\sim$ generated by $A=\Gamma(x, y)$ : i.e. if $(s, t)$ is in $A$ then so is $(t, s)$, and so on. Let $\Gamma_{t}$ denote the isotropy subgroup of $t$ in $\Gamma$.

LemMA 2. If $y \notin \Gamma x$ and $\Gamma_{x}=\Gamma_{y}$ then $a \sim b$ iff either $a=b$ or $(a, b) \in A$ or $(b, a) \in A$. Proof. If $a \neq b$ and $a \sim b$ then $a$ and $b$ lie in the $\Gamma$-orbit of either $x$ or $y$. Hence we assume that $a=x$. Now $x \sim b$ iff there exists a chain $z_{1}, \ldots, z_{n}$ such that

$$
\left(x, z_{1}\right) \in A \text { or }\left(z_{1}, x\right) \in A \text { and } z_{i} \sim z_{i+1} \text {. }
$$

If $\left(z_{1}, x\right) \in A$ then $x \in \Gamma y$, in contradiction to the assumptions. If $\left(x, z_{1}\right) \in A$ then there is a $\delta \in \Gamma$ s.t.

$$
\delta(x, y)=\left(x, z_{1}\right) .
$$

By the assumption on the isotropy groups

$$
y=z_{1} .
$$

The same reasoning applies to $y$, and the lemma is clear.

Suppose for the moment that $\alpha$ satisfies the assumptions of lemma 2. Then the quotient space of $B$ under $\sim$ is Hausdorff by the two lemmas, and clearly the action of $\Gamma$ on $B$ factors through $\sim$.

We have to see that the new action is not equivalent to the old one. The axial isometry $\gamma$ fixes two points on $B$ which are identified under $\sim$. Moreover, there are no new fixed points for $\gamma$, since otherwise there is an $s \in B$ such that

$$
(s, \gamma s)=\delta(x, y) \quad \text { or } \quad(\gamma s, s)=\delta(x, y)
$$

for some $\delta \in \Gamma$. In either case this implies that $y \in \Gamma x$.

To verify the assumptions in lemma 2 first assume that $\Gamma$ does not have torsion. [2, prop. 6.8] shows the equality of the isotropy groups (even if there is torsion). If $y=\delta x$ then $\delta \gamma \delta^{-1}$ has the geodesic through $(y, \delta y)$ as axis. On the other hand, $\gamma$ fixes $y$ and hence $\delta y$, by [2, prop. 6.8]. Since every non-elliptic isometry of $H$ has at most two fixed points in $B$ [2, prop. 6.5], $\delta$ permutes $x$ and $y$. If $\delta$ is non-elliptic, it has fixed points on the boundary, by the Brouwer fixed point theorem. So $\delta^{2}$ has at least three fixed points in $B$. In any case, $\delta^{2}$ is elliptic and hence is a torsion element, since an elliptic isometry in a lattice has finite order.

If $\Gamma$ has torsion not every axis $\alpha$ will satisfy the first assumption of lemma 2. But most $\alpha$ will do.

Consider a fundamental region $F$ for $\Gamma$ in $H$. The previous argument shows that, if $\delta x=y$, then $\delta^{2}$ is elliptic. Hence $\delta$ is a torsion element. In particular, $\delta^{2}$ will fix $\alpha$ pointwise. Since $\delta$ is orientation preserving, $\delta$ fixes $\alpha$ pointwise. By a conjugation 
we may assume that $\alpha$ goes through $F^{-}$. Since $F$ is a fundamental domain, $\alpha$ cannot pass through the interior of $F$. Therefore, an axis $\alpha$ that passes through the interior of $F$ satisfies the assumptions of lemma 2. Since the axes are dense in the geodesics [6, lemma $\left.8.3^{\prime}\right]$, such an axis will always exist. We obtain the following:

Proposition 1. Any lattice $\Gamma$ in a non-compact real rank 1 connected semi-simple Lie group $G$ with finite centre has a non-trivial (Hausdorff) quotient of the action on the boundary.

Proof. Consider the projection $p: G \rightarrow G^{\prime}$, where $G^{\prime}$ does not have compact factors. Then $p(\Gamma)$ is a lattice in $G^{\prime}$. So we can apply the construction above.

Proposition 1'. Let $M$ be a manifold of non-positive curvature whose universal cover $H$ satisfies: any two boundary points are joined by a unique geodesic. Then there always exists a non-trivial (Hausdorff) quotient of the action of $\pi_{1}(M)$ on the boundary.

Proof. In the construction above we only used [2, props. 6.5, 6.8] which hold for $\pi_{1}(M)$ for $M$ satisfying our conditions (cf. [2]). Since $\pi_{1}(M)$ does not have torsion (cf. [5, cor. 19.3]), any closed geodesic gives rise to a quotient.

II. SL $(n, \mathbb{Z})$ acting on $\mathbb{P}^{n-1}$

Let $\Gamma=\operatorname{SL}(n, \mathbb{Z})$ for short.

Proposition 2. All (Hausdorff) quotients of $\Gamma$ acting on $\mathbb{P}^{n-1}, n>2$, are trivial.

We first observe:

LeMMA 3. Let a group $\Gamma$ act on a compact Hausdorff space $M$. If the diagonal action of $\Gamma$ on $M \times M$-diagonal is minimal, then all equivariant $\Gamma$-quotients of $M$ are trivial. (Recall that an action of a group is called minimal if every orbit is dense.) Proof. Let $Y$ be such a quotient and let $C \subset C(M)=$ continuous functions on $M$ be the pullback of $C(Y)$ to $M$. Then $C$ is $\Gamma$-invariant. If $C \neq$ constants, pick $f \in C$ such that

$$
f\left(x_{0}\right) \neq f\left(y_{0}\right)
$$

for some $x_{0}, y_{0}$ in $M$. Pick neighbourhoods $U, V$ of $x_{0}, y_{0}$ such that $f(U)$ is disjoint from $f(V)$. If $x_{1} \neq y_{1}$ is any other pair of points in $M$, there exists a $\gamma \in \Gamma$ such that

$$
\gamma x_{1} \in U \text { and } \gamma y_{1} \in V .
$$

Hence

$$
f\left(x_{1}\right) \neq f\left(y_{1}\right)
$$

and $C$ separates points. By Stone-Weierstrass

$$
C=C(M) \text {. }
$$

Gelfand duality yields

$$
\boldsymbol{Y}=\boldsymbol{M} \text {. }
$$


Proposition 2 follows from the stronger:

Proposition 3. $\Gamma$ acts minimally on $\mathbb{P}^{n-1} \times \mathbb{P}^{n-1}$-diagonal.

Proof. First, on the level of $\mathbb{P}^{n-1}$ itself, we have:

LEMMA 4. $\Gamma$ acts minimally on $\mathbb{P}^{n-1}, n>1$.

Note. This is completely general: i.e. any lattice $\Gamma$ in a semi-simple Lie group $G$ without compact factors acts minimally on $G / P, P$ any parabolic (cf. [6, lemma 8.5]). Of course, the case at hand is standard and follows from elementary arguments.

Now the proof of proposition 3 develops in two stages. For notation let $\bar{x}$ be the line through $x$ for any $x \in \mathbb{R}^{n}$.

(1) Let $e_{i}$ be the standard basis of $\mathbb{R}^{n}$. Let $x \in \mathbb{R}^{n}, \bar{x} \neq \bar{e}_{1}$. We claim that $\Gamma\left(\bar{e}_{1}, \bar{x}\right)$ is dense in $\mathbb{P}^{n-1} \times \mathbb{P}^{n-1}$-diagonal. The stabilizer subgroup $\Gamma_{0}$ of $\Gamma$ at $\bar{e}_{1}$ looks like

$$
\left(\begin{array}{ccc}
* * & \cdots & * \\
0 * & \cdots & * \\
\cdot & \cdots & \cdot \\
0 * & \cdots & *
\end{array}\right) .
$$

In particular, embed SL $(n-1, \mathbb{Z})$ into $\Gamma_{0}$ in the obvious way. Clearly, it suffices to prove that $\Gamma\left(\bar{e}_{1}, \bar{y}\right)$ is dense in $\mathbb{P}^{n-1} \times \mathbb{P}^{n-1}$-diagonal for any $\bar{y}$ in the closure of $\Gamma_{0}(\bar{x})$. By lemma 4 and the above, we may assume that the coordinates $x_{2}, \ldots, x_{n}$ of $x$ are linearly independent over $Q$. Let $\bar{y} \neq \bar{z}$ be two lines and $V, W$ neighbourhoods of them. By lemma 4 there is a $\gamma \in \Gamma$ such that $\gamma\left(\bar{e}_{1}\right)$ is in $W$. Hence it suffices to find $\gamma_{0}$ such that $\gamma_{0}(\bar{x}) \in \gamma^{-1}(V)$. Let $\bar{t}=\gamma^{-1}(\bar{y})$. We can find $\gamma_{1} \in \operatorname{SL}(n-1, \mathbb{Z})$ such that

$$
\gamma_{1}\left(\overline{0, x_{2}, \ldots, x_{n}}\right)
$$

is close to

$$
\left(\overline{0, t_{2}, \ldots, t_{n}}\right)
$$

by lemma 4 . Let $x_{2}^{\prime}, \ldots, x_{n}^{\prime}$ be coordinates of

$$
\gamma_{1}\left(\overline{0, x_{2}, \ldots, x_{n}}\right)
$$

and pick coordinates $t_{i}$ for $\bar{t}$ such that $t_{i}$ is close to $x_{i}^{\prime}$ for $i>1$. The $x_{2}^{\prime}, \ldots, x_{n}^{\prime}$ are clearly linearly independent over $\mathbb{Q}$. The group generated by them is dense in $\mathbb{R}$ and we can find

$$
\gamma_{2}=\left(\begin{array}{lll}
1 m_{2} & \cdots & m_{n} \\
0 & & \\
\vdots & \text { id } & \\
0 & &
\end{array}\right) \in \Gamma_{0}
$$

such that $x_{1}^{\prime}+m_{2} x_{2}^{\prime}+\cdots m_{n} x_{n}^{\prime}$ is close to $t_{1}$. Since $\gamma_{2}$ leaves the other coordinates alone we have finished.

(2) Consider any two lines $\bar{y} \neq \bar{z}$. We claim that the closure of their $\Gamma$-orbit contains $\left(\bar{x}, \bar{e}_{1}\right)$ or $\left(\bar{e}_{1}, \bar{x}\right)$. We consider two cases:

(a) $\bar{z}$ is rational. Then we have the well-known result: 
LEMMA 5. $\Gamma \bar{e}_{1}=$ rational lines (i.e. all coprime $n$-tuples of integers lie in $\Gamma(1,0, \ldots)$ ). Proof. For $n=2$ this is clear. For $n>2$ let $\left(m_{1}, \ldots, m_{n}\right)$ be a point on a given line $l$ with integer entries. Then $l$ lies in the plane spanned by

$$
\left(m_{1}, \ldots, m_{n-1}, 0\right) \text { and } \bar{e}_{n} \text {. }
$$

By induction, pick $\gamma \in \mathrm{SL}(n-1, \mathbb{Z})$ such that

$$
\gamma \bar{e}_{1}=\left(m_{1}, \ldots, m_{n-1}, 0\right) \text {. }
$$

Then $\gamma^{-1}(l)$ lies in the plane spanned by $\bar{e}_{1}$ and $\bar{e}_{n}$ and we can use the case $n=2$.

(b) $\bar{z}$ is irrational. Then there are $i, j$ such that $z_{i}$ and $z_{j}$ are rationally independent, say $i=2, j=3$. In particular, $\mathbb{Z} z_{2}+\mathbb{Z} z_{3}$ is dense in $\mathbb{R}$. Hence there are matrices

$$
\gamma_{n}=\left(\begin{array}{lll}
1 m_{2}^{n} m_{3}^{n} 0 & \cdots & 0 \\
0 & & \\
\vdots & \text { id } &
\end{array}\right) \in \Gamma
$$

such that

$$
\gamma_{n} z \rightarrow\left(0, z_{2}, \ldots, z_{n}\right)
$$

as $n \rightarrow \infty$ while

$$
\gamma_{n} y=\left(1+m_{2}^{n} y_{2}+m_{3}^{n} y_{3}, y_{2}, y_{3}, \ldots\right) .
$$

If $\left.\overline{\left(z_{2}, z_{3}\right.}\right) \neq\left(\overline{y_{2}, y_{3}}\right)$, then

$$
\frac{m_{2}^{n} y_{2}+m_{3}^{n} y_{3}}{m_{2}^{n} z_{2}+m_{3}^{n} z_{3}}=\frac{y_{2}}{z_{2}}+m_{3}^{n} \frac{y_{3}-z_{3} y_{2} z_{2}^{-1}}{m_{2}^{n} z_{2}+m_{3}^{n} z_{3}} \rightarrow \pm \infty,
$$

since the denominator stays bounded and w.l.o.g.

$$
\left|m_{3}^{n}\right| \rightarrow+\infty \text {, }
$$

unless the slopes and so the lines are the same. We find that

$$
\gamma_{n} \bar{y} \rightarrow(\overline{1,0, \ldots})
$$

and we have finished.

If $\left(\overline{z_{2}, z_{3}}\right)=\left(\overline{y_{2}, y_{3}}\right)$, we can still pick $\gamma_{n}$ as above. Let

$$
a\left(z_{2}, z_{3}\right)=\left(y_{2}, y_{3}\right) \text {. }
$$

Then

$$
\gamma_{n} y \rightarrow\left(y_{1}-a z_{1}, y_{2}, \ldots\right)
$$

If

$$
\left(\overline{z_{1}, z_{2}, z_{3}}\right) \neq\left(\overline{y_{1}, y_{2}, y_{3}}\right)
$$

then

$$
y_{1}^{\prime} \stackrel{\text { def }}{=} y_{1}-a z_{1} \neq 0 \text {. }
$$

Notice that $y_{2}$ and $y_{3}$ are rationally independent, so one of $\left(y_{1}^{\prime}, y_{2}\right)$ or $\left(y_{3}^{\prime}, y_{3}\right)$ is rationally independent, say the first. Since

$$
\left(\overline{y_{1}^{\prime}, y_{2}}\right) \neq\left(\overline{0, z_{2}}\right)
$$


we can apply the previous argument to $\left(y_{1}^{\prime}, y_{2}\right)$. Instead of $z_{1}$ we could have used any $z_{i}, i>3$. We are left with the case

$$
\left(\overline{z_{2}, z_{3}, z_{i}}\right)=\left(\overline{y_{2}, y_{3}, y_{i}}\right)
$$

for all $i$, i.e.

$$
\bar{z}=\bar{y} .
$$

This is the final contradiction.

\section{REFERENCES}

[1] S. G. Dani \& S. Raghavan. Orbits of Euclidean frames under discrete linear groups. Israel J. Math. 36 (1980), 300-320.

[2] P. Eberlein \& B. O'Neil. Visibility manifolds. Pacific J. Math. 46 (1973), 45-109.

[3] G. A. Margulis. Quotient groups of discrete subgroups and measure theory. Func. Anal. Appl. 12 (1978), 295-305.

[4] G. A. Margulis. Finiteness of quotient groups of discrete subgroups. Func. Anal. Appl. 13 (1979), 178-187.

[5] J. Milnor. Morse Theory. Annals of Math. Studies no. 51. Princeton University Press: New Jersey, 1963.

[6] G. Mostow. Strong Rigidity of Locally Symmetric Spaces. Annals of Math. Studies no. 78. Princeton University Press: New Jersey, 1973.

[7] R. J. Zimmer. Equivariant images of projective space under the action of SL $(n, \mathbb{Z})$. Ergod. Th. and Dynam. Sys. 1 (1981), 519-522. 